\title{
Kinase Inhibitor Sensitivity
}

National Cancer Institute

\section{Source}

National Cancer Institute. Kinase Inhibitor Sensitivity. NCI Thesaurus. Code C126115.

A metabolic marker that predicts which kinase inhibitor agent could be an effective treatment for a patient's tumor based on the in vitro treatment response for cells extracted from the tumor. 